\title{
X/ur
}

\section{A Topological Approach for Automated Unstructured Meshing of Complex Reservoir}

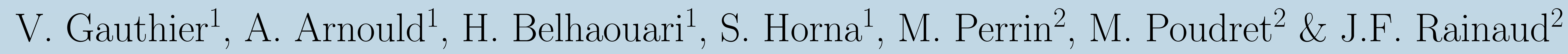 \\ 1 University of Poitiers, XLim, 2 Geosiris
}

\section{Objectives}

Sub-surface meshing for fluid-simulation.

Originality :

- Automated meshing based on geological interpretation

- Model consistency preserved

Image à insérer!
Topological based sub-surface model

Principle

- A topological structure includes

- Topological cells (vertices, edges, faces, volumes)

- Neighbor relationship between topological cells

Benefit :

- 1 rich model that contains all information : 2 geometries (the deposit domain and the actual domain), semantic labels (fault lips, surface name ...)

- 1 model mathematically defined that the consistency can be guaranteed

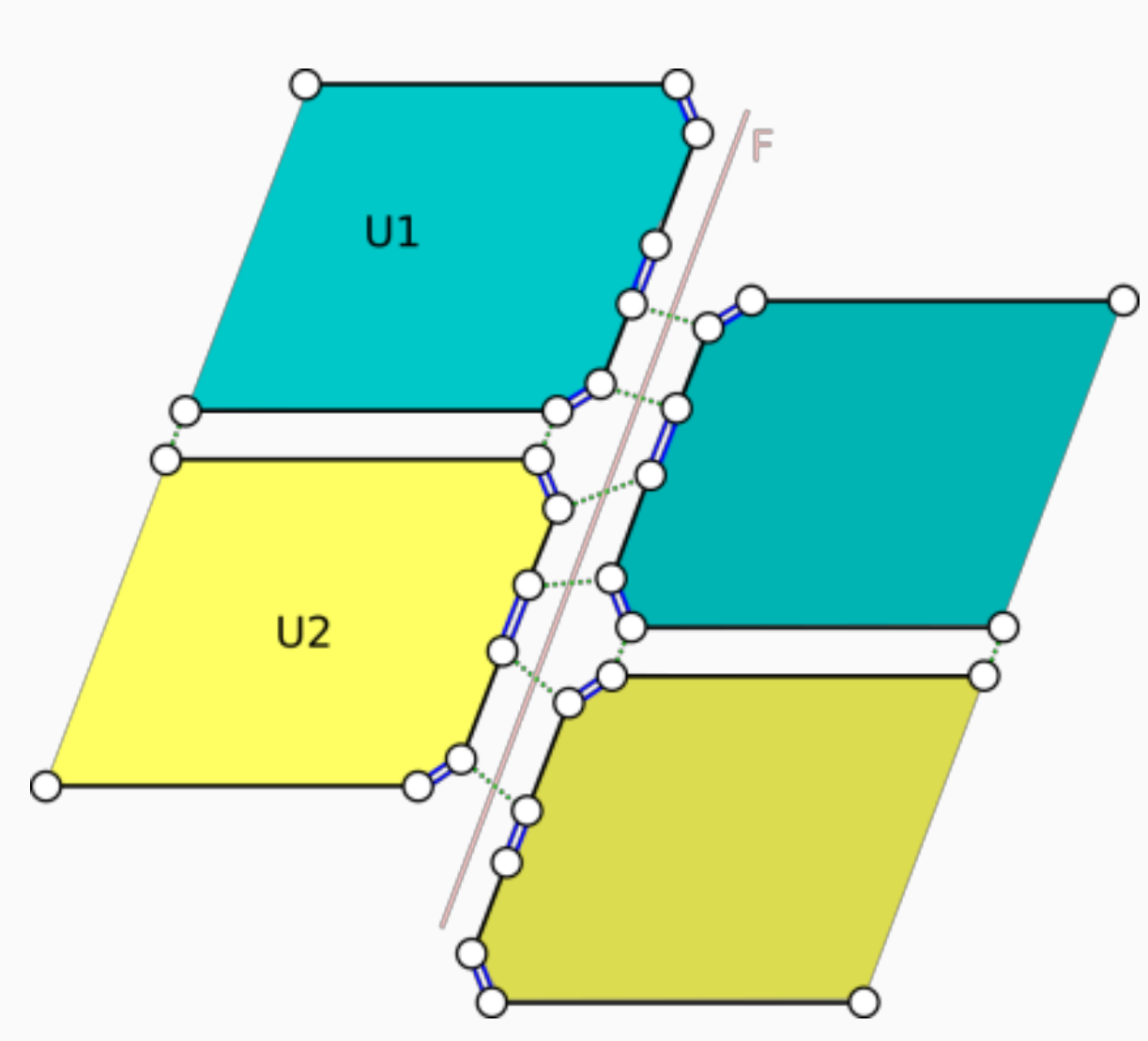

\section{Workflow}

\section{Structural part}

\section{Geological Interpretation}

The data collected by seismic and drilling survey are interpreted by geologist to determine the location of geological events (horizons, faults, onlap...). The RESQML standard is used to store all interpretations.

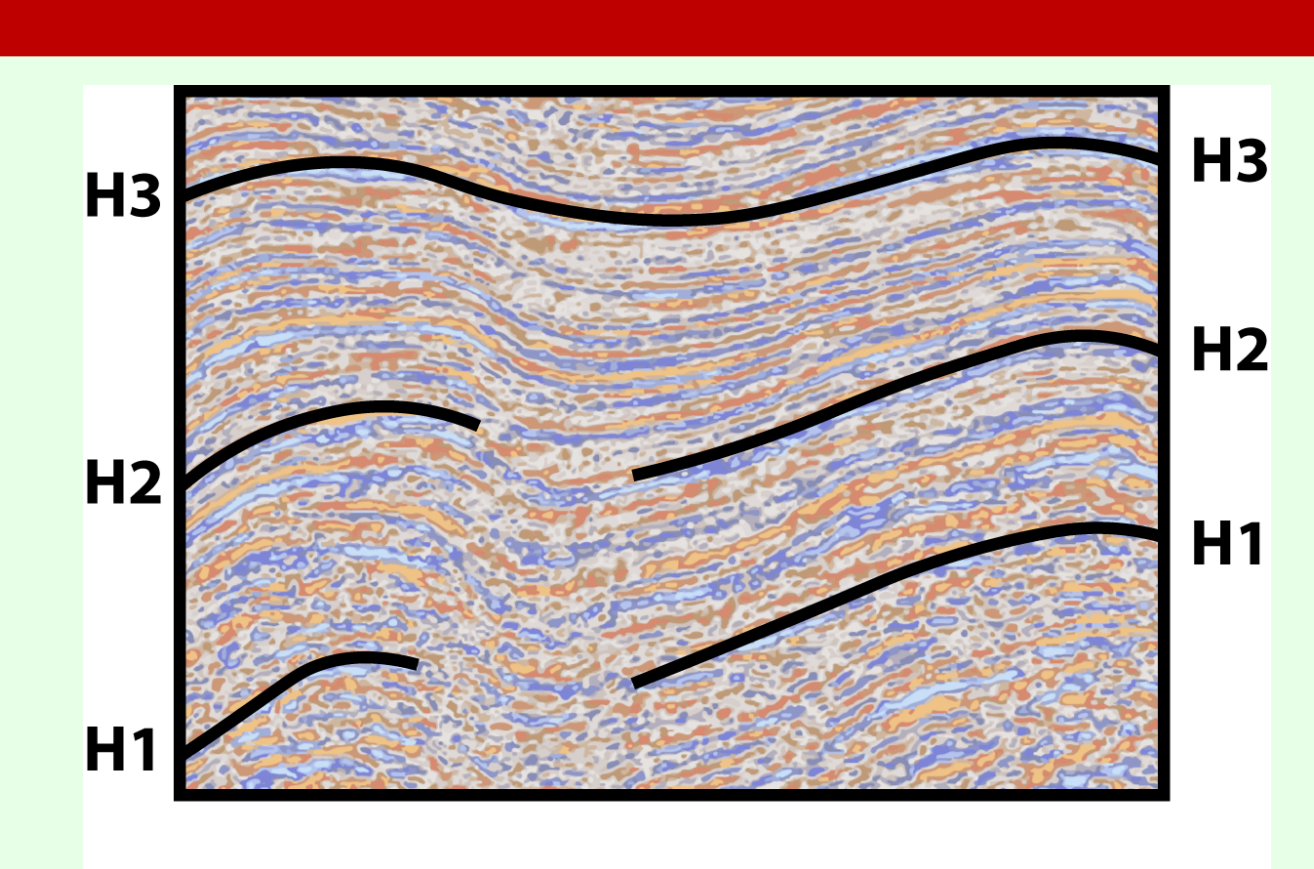

\section{D Meshing}

Linking top and bottom surfaces to compute 3D mesh, from the deposit domain. The fault position does not exist in the deposit domain, but it is necessary for the linking process. From the actual domain, the fault is interpolated in the deposit domain by using fault lips as control points.

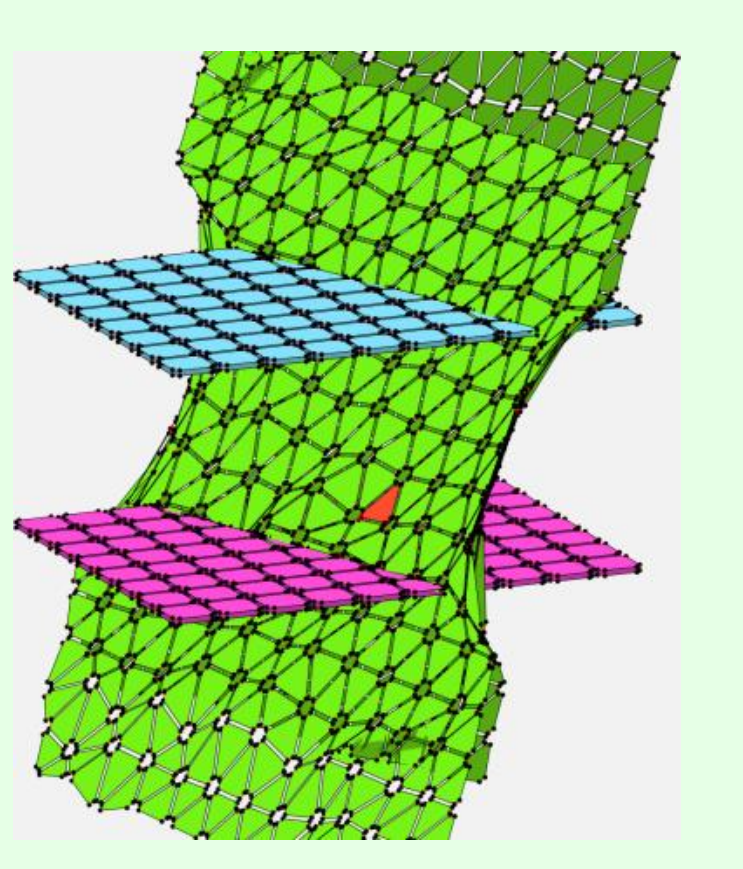

After the interpolation, the fault has a first geometry for the hanging wall, and a second for the footer wall. The topological model offers the possibility to keep topological relationship between both sides of the fault, with different geometry for both sides.

In the deposit space, the top and bottom are vertically linked far from faults. Near the faults, pillars extremities are delimited by faults. This extremities are interpolated to the actual domain.

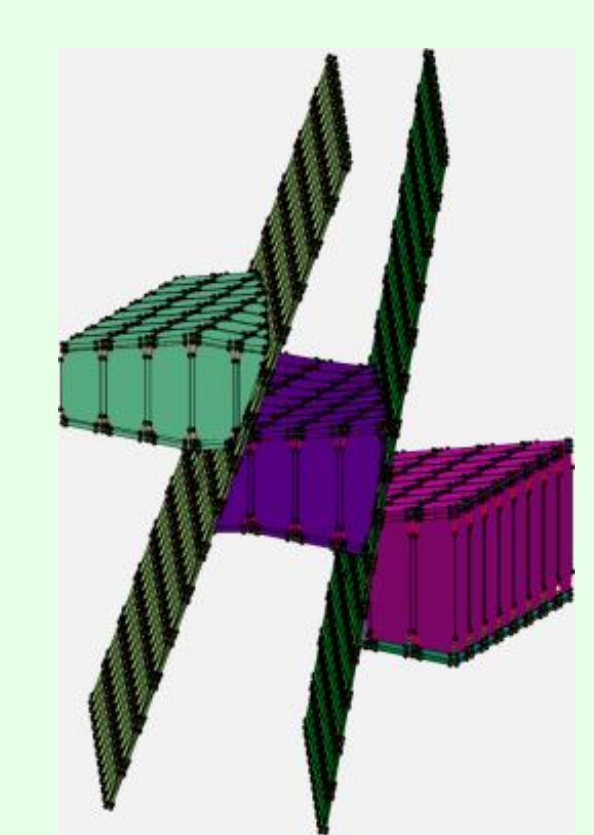

The Geosiris GeoTopoModeler prototype provides tools to specify the various contacts of the structural model (horizon/horizon and horizon/fault contacts) and to accordingly operate the necessary intersections and the removing of the parts with the "STOPS AT" process.

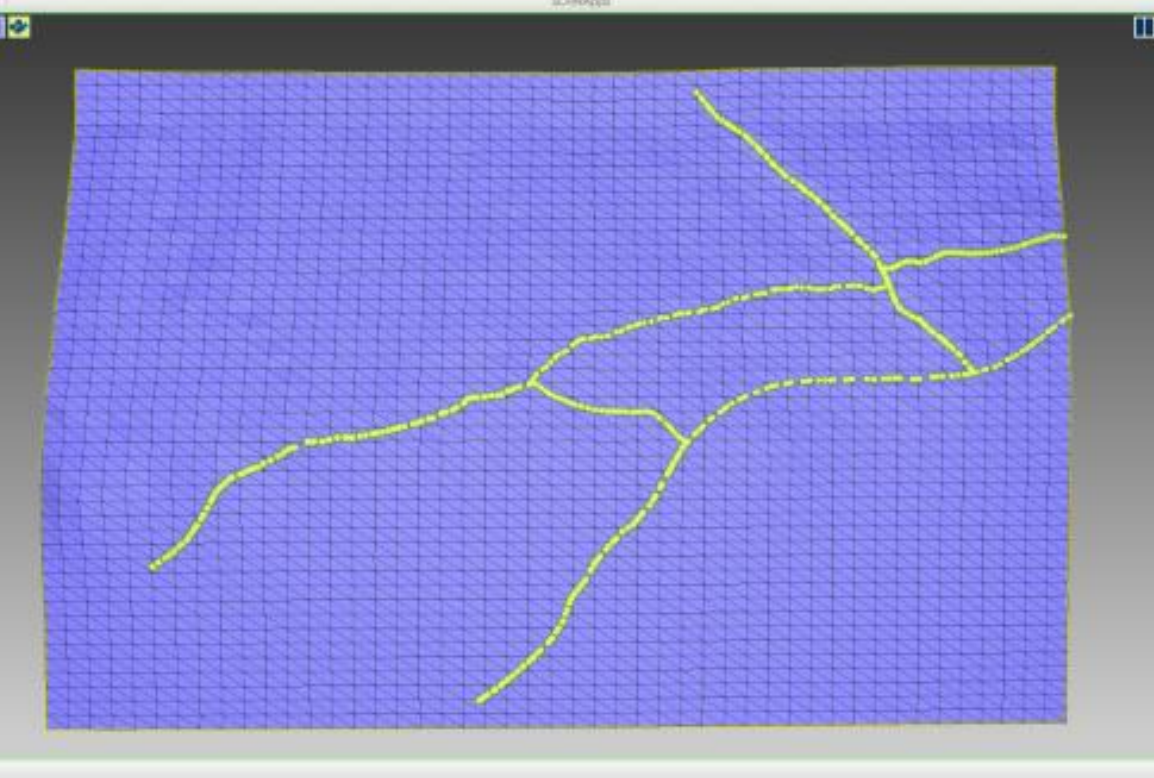

2DMeshing

A new quad surface is created based on structural surfaces. A regular grid is projected (in the deposit domain) on each horizon.

Advantage: The 2D mesh is the same for all surfaces (excepted at fault lips).
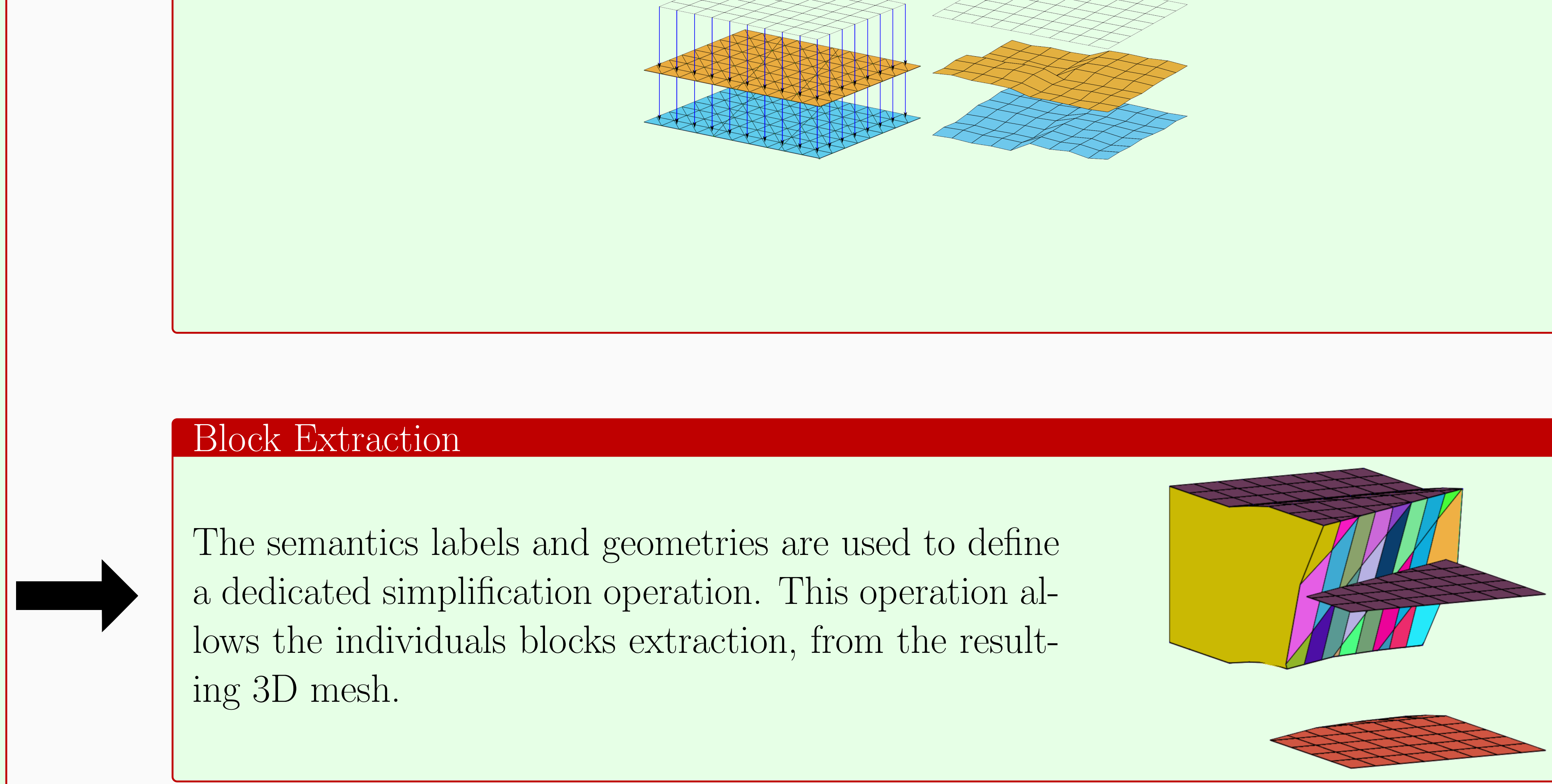

Results

\section{Conclusion}

The topological approach makes keeps the mesh consistency. Thus, with a single model, all information related to the mesh is stored in the same structure. It is also easy to extract information to work on these result by using the RESQML format.

\section{Future works}

To finalize the mesh, the blocks must be linked together. Each faces along faults must be splited to match faces of the oposit block. 